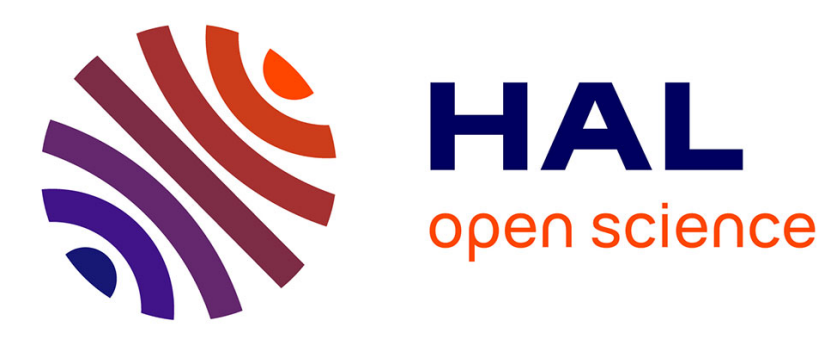

\title{
Flatness of Switched Linear Discrete-time Systems
}

Gilles Millérioux, Jamal Daafouz

\section{To cite this version:}

Gilles Millérioux, Jamal Daafouz. Flatness of Switched Linear Discrete-time Systems. IEEE Transactions on Automatic Control, 2009, 54 (3), pp.615-619. 10.1109/TAC.2008.2009589 . hal-00374998

\section{HAL Id: hal-00374998 https://hal.science/hal-00374998}

Submitted on 10 Apr 2009

HAL is a multi-disciplinary open access archive for the deposit and dissemination of scientific research documents, whether they are published or not. The documents may come from teaching and research institutions in France or abroad, or from public or private research centers.
L'archive ouverte pluridisciplinaire HAL, est destinée au dépôt et à la diffusion de documents scientifiques de niveau recherche, publiés ou non, émanant des établissements d'enseignement et de recherche français ou étrangers, des laboratoires publics ou privés. 


\title{
Flatness of Switched Linear Discrete-time
}

\section{Systems}

\author{
Gilles Millérioux, Jamal Daafouz \\ Nancy University, CNRS (France) \\ Centre de Recherche en Automatique de Nancy (CRAN), \\ gilles.milleriouxdesstin.uhp-nancy.fr \\ jamal.daafouz@ensem.inpl-nancy.fr
}

\begin{abstract}
This note is devoted to flatness for switched linear discrete-time systems. For this class of hybrid systems, algebraic conditions are derived to check whether a given output is flat. Then, a feedforward flatness-based control strategy for trajectory tracking is proposed.
\end{abstract}

\section{Index Terms}

Switched discrete-time systems, flatness, invertibility

\section{INTRODUCTION}

In this paper, we address the problem of fht output characterization and feedforward fhtnessbased control for switched multivariable linear discrete-time systems. Flatness was introduced by Fliess and al. [3] in 1995 and a deep insight can be found in the quite recent book [7]. Flatness-based control has been involved in many applications and has a compelling interest because it is well appropriate for, to mention a few, predictive control, trajectory planning, constraints handling. From the recent literature, it turns out that fhtness-based control can be especially attractive for switched linear systems as well. For instance [4] and references therein are interesting papers dedicated to the control of power converters. However, for switched systems, fhtness-based control still remains an open and challenging problem. Indeed, for linear systems, fhtness is merely equivalent to controllability and fat outputs can be easily characterized 
by some so-called defining matrices (see [5]). On the other hand, such a characterization is no longer valid for switched systems. A simple but special situation occurs when each linear mode of the switched system is active during a sufficient large time, in others words when a minimum "dwell-time" is guaranteed. In such a case, we can check for flat outputs for each linear models and one can then design a hybrid fatness-based control that takes care of each local dynamics. However, that amounts not only to discard the hybrid aspect but also this strategy may fail if the minimum "dwell-time" is not guaranteed. In this note, we relax the dwell-time constraint and thus we really consider the hybrid issue. We derive conditions which enable to check whether a given output of a switched linear discrete-time system is fht and a fhtness-based control strategy for trajectory tracking is suggested.

Notation : For any integer $l, \mathbf{1}_{l}$ refers to the $l$-dimensional identity matrix and $\mathbf{O}_{l \times l^{\prime}}$ stands for the $l \times l^{\prime}$ zero matrix. If irrelevant, the dimension of the zero matrix will be omitted and we shall merely write $\mathrm{O}$. For a matrix $X, X^{T}$ stands for transposition. $X^{\dagger}$ stands for the Moore-Penrose generalized inverse of $X$, namely the matrix of the same dimension as $X^{T}$ so that $X X^{\dagger} X=X$, $X^{\dagger} X X^{\dagger}=X^{\dagger}, X X^{\dagger}$ and $X^{\dagger} X$ are Hermitian.

\section{PREliminaries AND DEFINITIONS}

We examine switching linear discrete-time systems of the form

$$
\left\{\begin{array}{l}
x_{k+1}=A_{\sigma(k)} x_{k}+B_{\sigma(k)} u_{k} \\
y_{k}=C_{\sigma(k)} x_{k}+D_{\sigma(k)} u_{k}
\end{array}\right.
$$

where $x_{k} \in \mathbb{R}^{n}, u_{k} \in \mathbb{R}^{m}$ and $y_{k} \in \mathbb{R}^{p}$ are the states, the inputs and the measurements, respectively. All the matrices, namely $A_{\sigma(k)} \in \mathbb{R}^{n \times n}, B_{\sigma(k)} \in \mathbb{R}^{n \times m}, C_{\sigma(k)} \in \mathbb{R}^{p \times n}$ and $D_{\sigma(k)} \in$ $\mathbb{R}^{p \times m}$ belong to the respective finite sets $\left(A_{j}\right)_{1 \leq j \leq J},\left(B_{j}\right)_{1 \leq j \leq J},\left(C_{j}\right)_{1 \leq j \leq J}$ and $\left(D_{j}\right)_{1 \leq j \leq J}$. At a given time $k$, the index $j$ corresponds to the mode of the system and results from a switching function $\sigma: k \in \mathbb{N} \mapsto j=\sigma(k) \in\{1, \ldots, J\} .\{\sigma\}_{k_{1}}^{k_{1}+T}$ refers to the mode sequence $\left\{\sigma\left(k_{1}\right), \ldots, \sigma\left(k_{1}+T\right)\right\}$. For a given switching rule $\sigma$, the set of corresponding mode sequences over any interval of time of length $T+1$ is denoted by $\Sigma^{T}$. This set may contain either all possible mode sequences (also called paths) if there are not any repetitive switching patterns or can be reduced if any. We assume that the mode is known, either accessible or reconstructed (see [1] for this reconstruction issue). A key point lies in that no restriction on the time separation 
between switches ("dwell time") must be imposed.

Let $\mathcal{U}$ be the space of input sequences over $[0, \infty)$ and $\mathcal{Y}$ the corresponding output space. At time $k$, for each initial state $x_{k} \in \mathbb{R}^{n}$, when the system (1) is driven by the input sequence $\{u\}_{k}^{k+T}=\left\{u_{k}, \ldots, u_{k+T}\right\} \in \mathcal{U}$, for a mode sequence $\{\sigma\}_{k}^{k+T},\left\{x\left(x_{k}, \sigma, u\right)\right\}_{k}^{k+T}$ refers to the solution in the interval of time $[k, k+T]$ of (1) emanating from $x_{k}$ and $\left\{y\left(x_{k}, \sigma, u\right)\right\}_{k}^{k+T} \in \mathcal{Y}$ refers to the corresponding output sequence in the same interval of time $[k, k+T]$.

We introduce the subsequent vectors and matrices.

$$
\begin{gathered}
\text { For } i<0 \quad M_{\sigma(k)}^{i}=\mathbf{0}, \text { for } i=0 \quad M_{\sigma(k)}^{0}=D_{\sigma(k)} \text { and for } i>0, \\
M_{\sigma(k)}^{i}=\left(\begin{array}{ccccc}
D_{\sigma(k)} & \mathbf{0}_{p \times m} & \ldots & \ldots & \ldots \\
C_{\sigma(k+1)} B_{\sigma(k)} & D_{\sigma(k+1)} & \mathbf{0}_{p \times m} & \ldots & \ldots \\
\vdots & \vdots & \ddots & \ddots & \ddots \\
\vdots & \vdots & \ddots & \ddots & \ddots \\
C_{\sigma(k+i)} A_{\sigma(k+1)}^{\sigma(k+i-1)} B_{\sigma(k)} & C_{\sigma(k+i)} A_{\sigma(k+2)}^{\sigma(k+i-1)} B_{\sigma(k+1)} & \ldots & C_{\sigma(k+i)} B_{\sigma(k+i-1)} & D_{\sigma(k+i)}
\end{array}\right)
\end{gathered}
$$

with the transition matrix

$$
\begin{aligned}
& A_{\sigma\left(k_{0}\right)}^{\sigma\left(k_{1}\right)}=A_{\sigma\left(k_{1}\right)} A_{\sigma\left(k_{1}-1\right)} \ldots A_{\sigma\left(k_{0}\right)} \text { if } k_{1} \geq k_{0} \\
& =\mathbf{1}_{n} \text { if } k_{1}<k_{0} \\
& \bar{I}_{m}=\left(\begin{array}{ll}
\mathbf{1}_{m} & \mathbf{0}_{m \times(m \cdot r)}
\end{array}\right), \quad \bar{I}_{p}=\left(\begin{array}{ll}
\mathbf{0}_{p \times(p \cdot r)} & \mathbf{1}_{p}
\end{array}\right) \\
& \mathcal{O}_{\sigma(k)}^{i}=\left(\begin{array}{c}
C_{\sigma(k)} \\
C_{\sigma(k+1)} A_{\sigma(k)} \\
\vdots \\
C_{\sigma(k+i)} A_{\sigma(k)}^{\sigma(k+i-1)}
\end{array}\right), \mathcal{C}_{\sigma(k)}^{i}=\left(\begin{array}{llll}
A_{\sigma(k+1)}^{\sigma(k+i-1)} B_{\sigma(k)} & A_{\sigma(k+2)}^{\sigma(k+i-1)} B_{\sigma(k+1)} & \ldots & B_{\sigma(k+i-1)}
\end{array}\right) \\
& \underline{u}_{k}^{i}=\left(\begin{array}{c}
u_{k} \\
u_{k+1} \\
\vdots \\
u_{k+i}
\end{array}\right), \quad \underline{u}_{k}^{i}=\left(\begin{array}{c}
u_{k}^{\prime} \\
u_{k+1}^{\prime} \\
\vdots \\
u_{k+i}^{\prime}
\end{array}\right), \quad \underline{y}_{k}^{i}=\left(\begin{array}{c}
y_{k} \\
y_{k+1} \\
\vdots \\
y_{k+i}
\end{array}\right), \quad \underline{y}_{k}^{\prime}=\left(\begin{array}{c}
y_{k}^{\prime} \\
y_{k+1}^{\prime} \\
\vdots \\
y_{k+i}^{\prime}
\end{array}\right)
\end{aligned}
$$


When (1) is driven by an input sequence $\{u\}_{k}^{\infty} \in \mathcal{U}$ and a mode sequence $\{\sigma\}_{k}^{\infty}$, one has:

$$
\underline{y}_{k}^{i}=\mathcal{O}_{\sigma(k)}^{i} x_{k}+M_{\sigma(k)}^{i} \underline{u}_{k}^{i}
$$

Definition 1. When the system (1) is square $(m=p)$, it is said to be fht if there exists a set of independent variables $y_{k}$, referred to as flat outputs, such that all system variables can be expressed as a function of the flat outputs and a finite number of its backward and/or forward iterates. In particular, there exist two functions $\mathcal{F}$ and $\mathcal{G}$ which obey

$$
\left\{\begin{aligned}
x_{k} & =\mathcal{F}\left(y_{k+k_{\mathcal{F}}}, \ldots, y_{k+k_{\mathcal{F}}^{\prime}}\right) \\
u_{k} & =\mathcal{G}\left(y_{k+k_{\mathcal{G}}}, \ldots, y_{k+k_{\mathcal{G}}^{\prime}}\right)
\end{aligned}\right.
$$

where $k_{\mathcal{F}}, k_{\mathcal{F}}^{\prime}, k_{\mathcal{G}}$ and $k_{\mathcal{G}}^{\prime}$ are $\mathbb{Z}$-valued integers.

Both deriving algebraic conditions to check whether a given output of (1) is fhat and providing a feedforward fhtness-based control for trajectory tracking require a central notion namely the input invertibility.

\section{INPUT INVERTIBILITY}

We must distinguish input left and right invertibility. Some recent papers [8][6] have addressed the input left invertibility for switched discrete-time systems and the results are recalled in Subsect. III-A. In Subsect. III-B, we derive results on input right invertibility.

\section{A. Input left invertibility}

Definition 2. The system (1) is input left invertible if there exists a nonnegative integer $r<\infty$ such that, for all mode sequences in $\Sigma^{r}$, for two any inputs $\{u\}_{0}^{r},\left\{u^{\prime}\right\}_{0}^{r} \in \mathcal{U}$, the following implication applies:

$$
\left\{y\left(x_{0}, \sigma, u\right)\right\}_{0}^{r}=\left\{y\left(x_{0}, \sigma, u^{\prime}\right)\right\}_{0}^{r} \Rightarrow u_{0}=u_{0}^{\prime} \forall x_{0}
$$

In others words, by input left invertibility, we mean the ability to recover the input $u_{0}$ from a finite number of $r+1$ measurements $y_{i}(i=0, \ldots, r)$, the state vector $x_{0}$ at time $k=0$ and the mode sequences $\{\sigma\}_{0}^{r}$ being known. The term input has been introduced to emphasize the fact that only the input $u_{0}$ is expected to be recovered. The least integer $r$ for which (1) is input left invertible is called the left inherent delay or merely the delay. 
Theorem 1. The system (1) is input left invertible if there exists a nonnegative integer $r<\infty$ such that for all mode sequences in $\Sigma^{r}$,

$$
\operatorname{rank} M_{\sigma(k)}^{r}-\operatorname{rank} M_{\sigma(k+1)}^{r-1}=m
$$

Definition 3. A system is a left r-delay inverse for (1) if, under identical initial conditions $x_{0}$ and identical mode sequences $\{\sigma\}_{0}^{\infty}$, when driven by $\underline{y}_{k}^{r}$, its output fulfills $\hat{u}_{k+r}=u_{k}$ for all $k \geq 0$

Theorem 2. Assume that (1) is input left invertible with left inherent delay $r$. The system

$$
\left\{\begin{array}{l}
\hat{x}_{k+r+1}=P_{\sigma(k)}^{r} \hat{x}_{k+r}+B_{\sigma(k)} \bar{I}_{m} M_{\sigma(k)}^{r \dagger} \underline{y}_{k}^{r} \\
\hat{u}_{k+r}=-\bar{I}_{m} M_{\sigma(k)}^{r \dagger} \mathcal{O}_{\sigma(k)}^{r} \hat{x}_{k+r}+\bar{I}_{m} M_{\sigma(k)}^{r \dagger} \underline{y}_{k}^{r}
\end{array}\right.
$$

with

$$
P_{\sigma(k)}^{r}=A_{\sigma(k)}-B_{\sigma(k)} \bar{I}_{m} M_{\sigma(k)}^{r \dagger} \mathcal{O}_{\sigma(k)}^{r}
$$

is a left r-delay inverse system for (1).

Remark 1. In the Definition 2 and Definition 3, the initial condition is considered at the particular discrete time $k=0$ but can be replaced by any other initial condition $x_{k}$ taken at the discrete time $k$.

Remark 2. It is also shown that the state vector of the left $r$-delay inverse (10) fulfills $\hat{x}_{k+r}=x_{k}$ for all $k \geq 0$

\section{B. Input right invertibility}

Let us define a reference trajectory as a sequence of desired outputs $\left\{\tilde{y}_{k}, \tilde{y}_{k+1}, \ldots\right\}$ for $k \geq 0$.

Definition 4. The system (1) is input right invertible if there exists a nonnegative integer $r^{\prime}<\infty$ such that, for all mode sequences in $\Sigma^{r^{\prime}}$, for any reference output $\tilde{y}_{r^{\prime}}$ at time $k=r^{\prime}$ and for the initial condition $x_{0}=0$, there exists an input segment $\{u\}_{0}^{r^{\prime}}$ such that

$$
\left\{y\left(x_{0}=0, \sigma, u\right)\right\}_{0}^{r^{\prime}}=\{0\}_{0}^{r^{\prime}-1} * \tilde{y}_{r^{\prime}}
$$

where $\{0\}_{0}^{r^{\prime}-1} * \tilde{y}_{r^{\prime}}$ denotes a sequence of null vectors of dimension $p$ over the interval of time $\left[0, \ldots, r^{\prime}-1\right]$ and $\tilde{y}_{r^{\prime}}$ corresponds to the reference output at time $k=r^{\prime}$. The term input 
has been introduced as well to emphasize the fact that we are interested in finding out the input segment $\{u\}_{0}^{r^{\prime}}$ only. The least integer $r^{\prime}$ for which (1) is input right invertible will be called the right inherent delay or merely the delay.

Definition 5. A system, with input $\underline{y}_{k}^{r^{\prime}}$ is a right inverse for (1) if, under an identical initial condition $x_{0}$ and identical mode sequences $\{\sigma\}_{0}^{\infty}$, it can drives (1) such that $y_{k+r^{\prime}}=\tilde{y}_{k+r^{\prime}}$ for all $k \geq 0$

Remark 3. Similarly to the Remark 1, in the Definition 4 and Definition 5, the initial condition is considered at the particular discrete time $k=0$ but can be replaced by any other initial condition $x_{k}$ taken at the discrete time $k$.

Theorem 3. The following statements are equivalent.

i) The system (1) is input right invertible

ii) There exists a nonnegative integer $r^{\prime}<\infty$ such that, for all mode sequences in $\Sigma^{r^{\prime}}$, the equation with unknown $Q_{\sigma(k)}^{r^{\prime}}$

$$
M_{\sigma(k)}^{r^{\prime}} Q_{\sigma(k)}^{r^{\prime}}=\bar{I}_{p}^{T}
$$

has a solution

iii) There exists a nonnegative integer $r^{\prime}<\infty$ such that for all mode sequences in $\Sigma^{r^{\prime}}$,

$$
\operatorname{rank}\left(\begin{array}{cc}
\bar{I}_{p}^{T} & M_{\sigma(k)}^{r^{\prime}}
\end{array}\right)-\operatorname{rank} M_{\sigma(k)}^{r^{\prime}}=0
$$

iv) There exists a nonnegative integer $r^{\prime}<\infty$ such that for all mode sequences in $\Sigma^{r^{\prime}}$,

$$
\operatorname{rank} M_{\sigma(k)}^{r^{\prime}}-\operatorname{rank} M_{\sigma(k)}^{r^{\prime}-1}=p
$$

Proof: $i) \Leftrightarrow$ ii) On the interval of time $\left[k, k+r^{\prime}\right]$, according to (6), one has

$$
\underline{y}_{k}^{r^{\prime}}=\mathcal{O}_{\sigma(k)}^{r^{\prime}} x_{k}+M_{\sigma(k)}^{r^{\prime}} \underline{u}_{k}^{r^{\prime}}
$$

Besides, according to the Definition 4 of right invertibility, it is assumed that $x_{k}=0$ at time $k$. Thus, from (12) and (16) one has

$$
\underline{y}_{k}^{r^{\prime}}=M_{\sigma(k)}^{r^{\prime}} \underline{u}_{k}^{r^{\prime}}=\bar{I}_{p}^{T} \tilde{y}_{k+r^{\prime}}
$$


Input right invertibility means that this equality must be fulfilled for any $\tilde{y}_{k+r^{\prime}}$. As a result, the existence of $\underline{u}_{k}^{r^{\prime}}$ is strictly equivalent to the existence a matrix $Q_{\sigma(k)}^{r^{\prime}}$ fulfilling

$$
M_{\sigma(k)}^{r^{\prime}} Q_{\sigma(k)}^{r^{\prime}}=\left(\mathbf{0}_{p \times(p \cdot r)} \mathbf{1}_{p}\right)^{T}
$$

The matrix $Q_{\sigma(k)}^{r^{\prime}}$ reads explicitly $Q_{\sigma(k)}^{r^{\prime}}=M_{\sigma(k)}^{r^{\prime} \dagger} \bar{I}_{p}^{T}$

ii) $\Leftrightarrow$ iii) The relation (14) is the immediate consequence of the algebraic result stating that for any pairs $(W, Z)$ of matrices, the equation $W X=Z$ with unknown $X$ has a solution if and only if $\operatorname{rank}(W Z)=\operatorname{rank} W$.

iii) $\Leftrightarrow i v)$ We first notice that

$$
M_{\sigma(k)}^{r^{\prime}}=\left(\begin{array}{cc}
M_{\sigma(k)}^{r^{\prime}-1} & \mathbf{0}_{\left(p \cdot r^{\prime}\right) \times m} \\
C_{\sigma\left(k+r^{\prime}\right)} \mathcal{C}_{\sigma(k)}^{r^{\prime}} & D_{\sigma\left(k+r^{\prime}\right)}
\end{array}\right)
$$

Consequently,

$$
\left(\begin{array}{cc}
\bar{I}_{p}^{T} & M_{\sigma(k)}^{r^{\prime}}
\end{array}\right)=\left(\begin{array}{ccc}
\mathbf{0}_{\left(p \cdot r^{\prime}\right) \times p} & M_{\sigma(k)}^{r^{\prime}-1} & \mathbf{0}_{\left(p \cdot r^{\prime}\right) \times m} \\
\mathbf{1}_{p} & C_{\sigma\left(k+r^{\prime}\right)} \mathcal{C}_{\sigma(k)}^{r^{\prime}} & D_{\sigma\left(k+r^{\prime}\right)}
\end{array}\right)
$$

Hence

$$
\operatorname{rank}\left(\begin{array}{cc}
\bar{I}_{p}^{T} & M_{\sigma(k)}^{r^{\prime}}
\end{array}\right)=\operatorname{rank} M_{\sigma(k)}^{r^{\prime}-1}+\operatorname{rank}\left(\begin{array}{cc}
\mathbf{1}_{p} & D_{\sigma\left(k+r^{\prime}\right)}
\end{array}\right)
$$

Finally, noticing that $\operatorname{rank}\left(\begin{array}{cc}\mathbf{1}_{p} & D_{\sigma\left(k+r^{\prime}\right)}\end{array}\right)=p$, substituting the last equality into (14) gives $(15)$.

When (1) is input right invertible, we are looking for a second dynamical system which drives the system (1) so that its output tracks a reference trajectory $\tilde{y}_{k}, \tilde{y}_{k+1}, \ldots$ for $k \geq 0$. Let us define the following compound vector:

$$
\underline{y}_{k}^{i}=\left(\begin{array}{c}
\tilde{y}_{k} \\
\tilde{y}_{k+1} \\
\vdots \\
\tilde{y}_{k+i}
\end{array}\right)
$$

Theorem 4. Assume that (1) is input right invertible with right inherent delay $r^{\prime}$. The system

$$
\left\{\begin{array}{l}
\hat{x}_{k+1}=P_{\sigma(k)}^{r^{\prime}} \hat{x}_{k}+B_{\sigma(k)} \bar{I}_{m} M_{\sigma(k)}^{r^{\prime} \dagger} \underline{y}_{k}^{r^{\prime}} \\
u_{k}=-\bar{I}_{m} M_{\sigma(k)}^{r^{\prime} \dagger} \mathcal{O}_{\sigma(k)}^{r^{\prime}} \hat{x}_{k}+\bar{I}_{m} M_{\sigma(k)}^{r^{\prime} \dagger} \underline{y}_{k}^{r^{\prime}}
\end{array}\right.
$$


with

$$
P_{\sigma(k)}^{r^{\prime}}=A_{\sigma(k)}-B_{\sigma(k)} \bar{I}_{m} M_{\sigma(k)}^{r^{\prime} \dagger} \mathcal{O}_{\sigma(k)}^{r^{\prime}}
$$

is a right $r^{\prime}$-delay inverse system for (1).

Proof: Notice that

$$
\begin{aligned}
y_{k+r^{\prime}}-\tilde{y}_{k+r^{\prime}} & =\bar{I}_{p} \underline{y}_{k}^{r^{\prime}}-\tilde{y}_{k+r^{\prime}} \\
& =\bar{I}_{p}\left(\mathcal{O}_{\sigma(k)}^{r^{\prime}} x_{k}+M_{\sigma(k)}^{r^{\prime}} \underline{u}_{k}^{r^{\prime}}\right)-\tilde{y}_{k+r^{\prime}} \\
& =\bar{I}_{p} \mathcal{O}_{\sigma(k)}^{r^{\prime}} x_{k}-\bar{I}_{p} M_{\sigma(k)}^{r^{\prime}} M_{\sigma(k)}^{r^{\prime} \dagger} \mathcal{O}_{\sigma(k)}^{r^{\prime}} \hat{x}_{k}+\bar{I}_{p} M_{\sigma(k)}^{r^{\prime}} M_{\sigma(k)}^{r^{\prime} \dagger} \underline{y}_{k}^{r^{\prime}}-\tilde{y}_{k+r^{\prime}}
\end{aligned}
$$

Since (1) is input right invertible, (13) holds and is equivalent to

$$
M_{\sigma(k)}^{r^{\prime}} M_{\sigma(k)}^{r^{\prime} \dagger} \bar{I}_{p}^{T}=\bar{I}_{p}^{T}
$$

Therefore,

$$
\bar{I}_{p}\left(M_{\sigma(k)}^{r^{\prime}} M_{\sigma(k)}^{r^{\prime} \dagger}\right)^{T}=\bar{I}_{p}
$$

The Moore-Penrose generalized inverse being hermitian, one has

$$
\left(M_{\sigma(k)}^{r^{\prime}} M_{\sigma(k)}^{r^{\prime} \dagger}\right)^{T}=M_{\sigma(k)}^{r^{\prime}} M_{\sigma(k)}^{r^{\prime} \dagger}
$$

and the following equality applies

$$
\bar{I}_{p} M_{\sigma(k)}^{r^{\prime}} M_{\sigma(k)}^{r^{\prime} \dagger}=\bar{I}_{p}
$$

As a result, taking into account (18), $y_{k+r^{\prime}}-\tilde{y}_{k+r^{\prime}}$ finally reads

$$
y_{k+r^{\prime}}-\tilde{y}_{k+r^{\prime}}=\bar{I}_{p} \mathcal{O}_{\sigma(k)}^{r^{\prime}}\left(x_{k}-\hat{x}_{k}\right)
$$

On the other hand, it is easy to see that

$$
x_{k+1}-\hat{x}_{k+1}=A_{\sigma(k)}\left(x_{k}-\hat{x}_{k}\right)
$$

In view of the Definition 5, we must consider that (1) and (17) are initialized such that $\hat{x}_{0}=x_{0}$. By virtue of (20), we infer that $x_{k}-\hat{x}_{k}=0$ for all $k \geq 0$ and thus $y_{k+r^{\prime}}-\tilde{y}_{k+r^{\prime}}=0$ for all $k \geq 0$.

When the system (1) is both left and right invertible with left and right inherent delays $r=r^{\prime}$, it will be merely said that the system (1) is input invertible with inherent delay $r=r^{\prime}=R$. 
Remark 4. It is interesting to notice that when the system (1) is square, if $\Sigma^{r}=\Sigma^{r^{\prime}}$ and the sets contain the all possible mode sequences over any interval of time of length $r+1=r^{\prime}+1$, input left invertibility is equivalent to input right invertibility. Indeed, in such a case, (9) is equivalent to (15).

\section{Flatness}

\section{A. Flat output characterization}

Let us define the inverse transition matrix as

$$
\begin{aligned}
P_{\sigma\left(k_{0}\right)}^{\sigma\left(k_{1}\right)} & =P_{\sigma\left(k_{1}\right)}^{r} P_{\sigma\left(k_{1}-1\right)}^{r} \ldots P_{\sigma\left(k_{0}\right)}^{r} \text { if } k_{1} \geq k_{0} \\
& =\mathbf{1}_{n} \text { if } k_{1}<k_{0}
\end{aligned}
$$

with

$$
P_{\sigma(k)}^{r}=A_{\sigma(k)}-B_{\sigma(k)} \bar{I}_{m} M_{\sigma(k)}^{r \dagger} \mathcal{O}_{\sigma(k)}^{r}
$$

Theorem 5. A componentwise independent output $y_{k}$ of the system (1) assumed to be square $(m=p)$ and left input invertible with inherent delay $r$, is a fht output if there exists a positive integer $K<\infty$ such that, for all mode sequences in $\Sigma^{r+K-1}$, the following equality applies for all $k \geq 0$ :

$$
P_{\sigma(k)}^{\sigma(k+K-1)}=\mathbf{0}
$$

$\Sigma^{r+K-1}$ stands for the set of mode sequences over the interval of time $[k, \ldots, k+r+K-1]$.

Proof: Assume that the system (1) is input invertible with inherent delay $r$, thus the left $r$-delay inverse system (10) exists. Iterating (10) yields:

$$
\begin{aligned}
& \hat{x}_{k+r+l}=P_{\sigma(k)}^{\sigma(k+l-1)} \hat{x}_{k+r}+\sum_{i=0}^{l-1} P_{\sigma(k+i+1)}^{\sigma(k+l-1)} B_{\sigma(k+i)} \bar{I}_{m} M_{\sigma(k+i)}^{r \dagger} \underline{y}_{k+i}^{r} \\
& \hat{u}_{k+r+l}=\bar{I}_{m} M_{\sigma(k+l)}^{r \dagger}\left(\underline{y}_{k+l}^{r}-\mathcal{O}_{\sigma(k+l)}^{r} \hat{x}_{k+r+l}\right)
\end{aligned}
$$

If (21) is fulfilled with $l=K,(22)$ turns into:

$$
\begin{aligned}
& \hat{x}_{k+r+K}=\sum_{i=0}^{K-1} P_{\sigma(k+i+1)}^{\sigma(k+K-1)} B_{\sigma(k+i)} \bar{I}_{m} M_{\sigma(k+i)}^{r \dagger} \underline{y}_{k+i}^{r} \\
& \left.\hat{u}_{k+r+K}=\bar{I}_{m} M_{\sigma(k+K)}^{r \dagger} \underline{y}_{k+K}^{r}-\mathcal{O}_{\sigma(k+K)}^{r} \hat{x}_{k+r+K}\right)
\end{aligned}
$$

revealing that $\hat{x}_{k+r+K}$ and so $\hat{u}_{u+r+K}$ is independent of $\hat{x}_{k+r}$. In particular, (23) holds for $\hat{x}_{k_{0}+r}=x_{k_{0}}$ for all $k_{0} \geq 0$ which implies that $\hat{x}_{k+r+K}=x_{k+K}$ and $\hat{u}_{k+r+K}=u_{k+K}$ for all 
$k \geq 0$. Therefore, after performing the change of variable $k \rightarrow k-K$, we obtain

$$
x_{k}=\sum_{i=0}^{K-1} P_{\sigma(k+i+1-K)}^{\sigma(k-1)} B_{\sigma(k+i-K)} \bar{I}_{m} M_{\sigma(k+i-K)}^{r \dagger} \underline{y}_{k+i-K}^{r}
$$

and

$$
\left.u_{k}=\bar{I}_{m} M_{\sigma(k)}^{r \dagger} \underline{y}_{k}^{r}-\mathcal{O}_{\sigma(k)}^{r}\left(\sum_{i=0}^{K-1} P_{\sigma(k+i+1-K)}^{\sigma(k-1)} B_{\sigma(k+i-K)} \bar{I}_{m} M_{\sigma(k+i-K)}^{r \dagger} \underline{y}_{k+i-K}^{r}\right)\right)
$$

Equations (24) and (25) reveal that $x_{k}$ and $u_{k}$ can be expressed as a function of $y_{k}$ and a finite number of its backward and/or forward iterates in the form (7), that is to say $y_{k}$ is a flat output.

\section{B. Flatness-based control for trajectory tracking}

If we are interested in delivering the fht control (25) in a recursive way, the following theorem can be useful.

Theorem 6. Assume that (1) is (left and right) input invertible with inherent delay $R$. If there exists a positive integer $K<\infty$ such that for all mode sequences in $\Sigma^{R+K-1}$,

$$
P_{\sigma(k)}^{\sigma(k+K-1)}=\mathbf{0}
$$

with

$$
P_{\sigma(k)}^{R}=A_{\sigma(k)}-B_{\sigma(k)} \bar{I}_{m} M_{\sigma(k)}^{R \dagger} \mathcal{O}_{\sigma(k)}^{R}
$$

then the control $u_{k}$ delivered by

$$
\left\{\begin{array}{l}
\hat{x}_{k+1}=P_{\sigma(k)}^{R} \hat{x}_{k}+B_{\sigma(k)} \bar{I}_{m} M_{\sigma(k)}^{R \dagger} \underline{\tilde{y}}_{k}^{R} \\
u_{k}=\bar{I}_{m} \underline{u}_{k}^{R}=-\bar{I}_{m} M_{\sigma(k)}^{R \dagger} \mathcal{O}_{\sigma(k)}^{R} \hat{x}_{k}+\bar{I}_{m} M_{\sigma(k)}^{R \dagger} \underline{y}_{k}^{R}
\end{array}\right.
$$

is a flat control and guarantees a stable trajectory tracking whenever the system (20) is uniformly asymptotically stable (u.a.s).

Proof: On one hand, if (1) is input invertible with inherent delay $R$, by virtue of (19) and (20) in the proof of Theorem 4 , after substituting $r^{\prime}$ by $R$, since $\hat{x}_{0}$ may differ from $x_{0}$, one has 
$\lim _{k \rightarrow \infty} y_{k+R}-\tilde{y}_{k+R}=0$ whenever (20) is u.a.s. On the other hand, iterating (27) $K-1$ times yields

$$
\begin{aligned}
& \hat{x}_{k+K}=P_{\sigma(k)}^{\sigma(k+K-1)} \hat{x}_{k}+\sum_{i=0}^{K-1} P_{\sigma(k+i+1)}^{\sigma(k+K-1)} B_{\sigma(k+i)} \bar{I}_{m} M_{\sigma(k+i)}^{R \dagger} \underline{y}_{k+i}^{R} \\
& \left.u_{k+K}=\bar{I}_{m} M_{\sigma(k+K)}^{R \dagger} \underline{y}_{k+K}^{R}-\mathcal{O}_{\sigma(k+K)}^{R} \hat{x}_{k+K}\right)
\end{aligned}
$$

Finally, if (26) is fulfilled, the control $u_{k}$ reads:

$$
u_{k}=\bar{I}_{m} M_{\sigma(k)}^{R \dagger}\left(\underline{y}_{k}^{R}-\mathcal{O}_{\sigma(k)}^{R}\left(\sum_{i=0}^{K-1} P_{\sigma(k+i+1-K)}^{\sigma(k-1)} B_{\sigma(k+i-K)} \bar{I}_{m} M_{\sigma(k+i-K)}^{R \dagger} \underline{y}_{k+i-K}^{R}\right)\right)
$$

revealing that $u_{k}$ is a fht control since it only depends on the output.

The problem of assessing the stability of (20) turns into a usual switched stability analysis problem. We can resort to polyquadratic stability ([2]).

\section{Illustrative example}

We shall consider a MIMO switched linear discrete-time system of the form (1) and having two distinct modes $(J=2)$. The respective finite sets $\left(A_{j}\right)_{1 \leq j \leq 2},\left(B_{j}\right)_{1 \leq j \leq 2},\left(C_{j}\right)_{1 \leq j \leq 2}$ and $\left(D_{j}\right)_{1 \leq j \leq 2}$ are

$$
\begin{aligned}
& A_{1}=\left(\begin{array}{cccc}
0.3 & 1 & 0 & 1 \\
0.3 & 0 & 0.1 & 0.2 \\
-0.1 & 0 & 0 & -0.4 \\
0.01 & 0.1 & 0.2 & 0
\end{array}\right), \quad A_{2}=\left(\begin{array}{cccc}
0.3 & 2 & 0 & 1 \\
0.3 & 0 & 0.1 & 0.2 \\
-0.6 & 0 & 0 & -0.4 \\
0.01 & 0.1 & 0.2 & 0
\end{array}\right), \\
& B_{1}=B_{2}=\left(\begin{array}{cc}
-1 & 0.5 \\
0 & -1.5 \\
1 & 0 \\
0 & 1
\end{array}\right), C_{1}=C_{2}=\left(\begin{array}{llll}
1 & 1 & 1 & 1 \\
1 & 3 & 1 & 4
\end{array}\right), D_{1}=D_{2}=\mathbf{0}
\end{aligned}
$$

The system is driven by some inputs $\{u\}_{0}^{\infty} \in \mathcal{U}$, the set of input sequences ranging between two arbitrary bounds, say 0 and 1 for the example. We shall examine three distinct switching rules: a switching rule which obeys $\sigma(k)=1$ for all $k$ which amounts to consider a mere linear system (case 1), a switching rule which obeys $\sigma(k)=2$ for all $k$ which also amounts to consider 
a mere linear system (case 2), a switching rule for which $\sigma(k)$ alternates randomly between 1 and 2 , the occurrence of two consecutive modes 1 being however not allowed.

We first consider the case 1. It turns out that (9) and (15) hold for $r=r^{\prime}=2$. Hence, according to the Theorem 1 and Theorem 3, the system is left and right input invertible with inherent delay $r=r^{\prime}=2$. The set $\Sigma^{r}$ is merely composed of a single mode sequence $\{1,1,1\}$. According to the Theorem $5, y_{k}$ is a flat output because (21) is fulfilled with $K=2$. For the case 2 , the situation is exactly the same than the case 1 except that the set $\Sigma^{r}$ is composed of $\{2,2,2\}$. As a result, it can be stressed that the minimum dwell time equals 2 . Then, we consider the case 3 . Such a case is interesting in that the switching rule does not always guarantee the minimum dwell time. It turns out that (9) and (15) hold for $r=r^{\prime}=2$. Hence, according to Theorem 1 and Theorem 3, the system is input invertible with inherent delay $r=r^{\prime}=R=2$. The set $\Sigma^{R}$ does not contain all possible mode sequences but only the patterns $\{\{1,2,1\},\{1,2,2\},\{2,1,2\},\{2,2,1\},\{2,2,2\}\}$, the only ones which are compatible with the switching rule. Equation (26) is fulfilled with $K=2$. Consequently the assumptions of Theorem 6 hold and a feedforward fatness-based control can be designed.
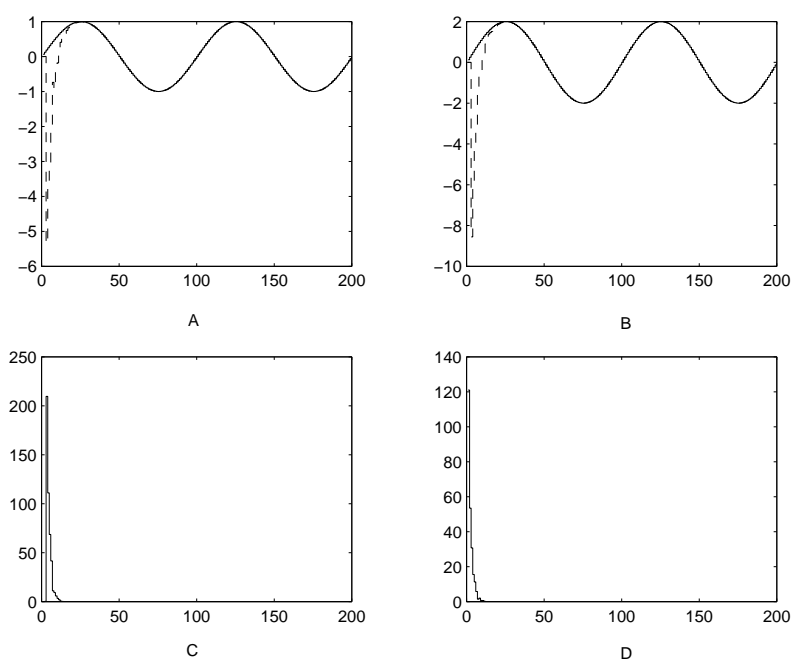

Fig. 1. A : solid line $\tilde{y}_{k}^{(1)}$ and dashed line $y_{k}^{(1)}$. B : solid line $\tilde{y}_{k}^{(2)}$ and dashed line $y_{k}^{(2)} \cdot \mathrm{C}:\left|y_{k}-\tilde{y}_{k}\right|^{2}$. D : $\left|x_{k}-\hat{x}_{k}\right|^{2}$

Two distinct sinusoidal trajectories $\left\{\tilde{y}_{k}^{(1)}\right\}_{0}^{\infty}$ and $\left\{\tilde{y}_{k}^{(2)}\right\}_{0}^{\infty}$ have been planified for the two respective entries $y_{k}^{(1)}$ and $y_{k}^{(2)}$ of the output $y_{k}$. It can be verified through a LMI approach ([2]) 
that (20) is u.a.s, more precisely, it can be checked that it is polyquadratically stable. Figure 1 represents the results of the trajectory tracking based on the fht control delivered by (27). The time plots illustrate that the desired trajectories are well tracked. The convergences toward zero are consistent with the theoretical results.

\section{CONCLUSION}

For switched linear discrete-time systems, algebraic conditions in terms of the state description matrices to check whether a given output is fht have been derived. Then, it has been shown how a feedforward fht control for trajectory tracking can be carried out in the case when a minimum dwell time is not guaranteed. Let us finally mention that one can resort to these conditions and to such a fht control when considering periodic systems or monovariate systems with time-varying relative degree.

Acknowledgement This work is partially funded by a grant from the Agence Nationale pour la Recherche in France (Ref. ANR-05-JCJC-0112-01)

\section{REFERENCES}

[1] A. Balluchi, L. Benvenuti, M. D. Di Benedetto, and A. L. Sangiovanni-Vincentelli. Design of Observers for Hybrid Systems, volume 2289 of Lecture Notes in Computer Science: Hybrid Systems: Computation and Control, pages 76-89. Springer-Verlag, Berlin Heidelberg New York, 2002.

[2] J. Daafouz, G. Millérioux, and C. Iung. A poly-quadratic stability based approach for switched systems. International Journal of Control, 75:1302-1310, November 2002.

[3] M. Fliess, J. Levine, P. Martin, and P. Rouchon. Flatness and defect of non-linear systems: introductory theory and examples. Int. Jour. of Control, 61(6):1327-1361, 1995.

[4] A. Gensior, O. Woywode, J. Rudolph, and H. Guldner. On differential fhtness, trajectory planning, observers, and stabilization for dc-dc converters. IEEE Trans. on Circuits and Systems - I Regular Papers, 53(9), September 2006.

[5] J. Levine and D. V. Nguyen. Flat output characterization for linear systems using polynomial matrices. Systems and control Letters, 48:69-75, 2003.

[6] G. Millérioux and J. Daafouz. Invertibility and fhtness of switched linear discrete-time systems. In Proc. of the 10th International Conference on Hybrid Systems: Computation and Control (HSCC'07), Pisa, Italy, April 2007.

[7] H. Sira-Ramirez and S. K. Agrawal. Differentially Flat Systems. Marcel Dekker, New York, 2004.

[8] S. Sundaram and C. Hadjicostis. Designing stable inverters and state observers for switched linear systems with unknown inputs. In Proc. of the 45th IEEE Conference on Decision and Control, San Diego, CA, USA, December 2006. 